\title{
Evaluation of a novel type of imaging probe based on a recombinant bivalent mini-antibody construct for detection of CD44v6-expressing squamous cell carcinoma
}

\author{
ANNA-KARIN HAYLOCK ${ }^{1,2^{*}}$, DIANA SPIEGELBERG ${ }^{1 *}$, ANJA C. MORTENSEN $^{1}$, RAM K. SELVARAJU ${ }^{3}$, \\ JOHAN NILVEBRANT ${ }^{4}$, OLOF ERIKSSON ${ }^{3}$, VLADIMIR TOLMACHEV ${ }^{1}$ and MARIKA V. NESTOR ${ }^{1,2}$ \\ ${ }^{1}$ Department of Immunology, Genetics and Pathology Uppsala University, Uppsala; \\ ${ }^{2}$ Unit of Otolaryngology and Head and Neck Surgery, Department of Surgical Sciences, Uppsala University, Uppsala; \\ ${ }^{3}$ Preclinical PET Platform, Department of Medicinal Chemistry, Uppsala University, Uppsala; \\ ${ }^{4}$ Division of Protein Technology, School of Biotechnology, Royal Institute of Technology, Stockholm, Sweden
}

Received August 19, 2015; Accepted October 31, 2015

DOI: 10.3892/ijo.2015.3290

\begin{abstract}
We have developed the CD44v6-targeting human bivalent antibody fragment AbD19384, an engineered recombinant human bivalent Fab antibody formed via dimerization of dHLX (synthetic double helix loop helix motif) domains, for potential use in antibody-based molecular imaging of squamous cell carcinoma in the head and neck region. This is a unique construct that has, to the best of our knowledge, never been assessed for molecular imaging in vivo before. The objective of the present study was to evaluate for the first time the in vitro and in vivo binding properties of radio-iodinated AbD19384, and to assess its utility as a targeting agent for molecular imaging of CD44v6-expressing tumors. Antigen specificity and binding properties were assessed in vitro. In vivo specificity and biodistribution of ${ }^{125} \mathrm{I}-\mathrm{AbD} 19384$ were next evaluated in tumor-bearing mice using a dual-tumor setup. Finally, AbD19384 was labeled with ${ }^{124}$ I, and its imaging properties were assessed by small animal PET/CT in tumor bearing mice, and compared with 2-deoxy-2-[18F]fluoro-D-glucose $\left({ }^{18} \mathrm{~F}-\mathrm{FDG}\right)$. In vitro studies demonstrated CD44v6-specific binding with slow off-rate for AbD19384. A favorable biodistribution profile was seen in vivo, with tumor-specific uptake. Small animal PET/CT images of ${ }^{124} \mathrm{I}-\mathrm{AbD} 19384$ supported the results through clearly visible high CD44v6-expressing tumors and faintly visible low expressing tumors, with superior imaging properties compared to ${ }^{18} \mathrm{~F}$-FDG. Tumor-to-blood
\end{abstract}

Correspondence to: Dr Marika V. Nestor, Department of Immunology, Genetics and Pathology, The Rudbeck Laboratory, SE-75185 Uppsala, Sweden

E-mail: marika.nestor@igp.uu.se

*Contributed equally

Key words: molecular imaging, CD44v6, Fab-dHLX, F(ab') ${ }_{2}$, recombinant antibodies, immuno-PET, head and neck squamous cell carcinoma ratios increased with time for the conjugate (assessed up to $72 \mathrm{~h}$ p.i.), although $48 \mathrm{~h}$ p.i. proved best for imaging. Biodistribution and small-animal PET studies demonstrated that the recombinant Fab-dHLX construct AbD19384 is a promising tracer for imaging of CD44v6 antigen expression in vivo, with the future aim to be used for individualized diagnosis and early detection of squamous cell carcinomas in the head and neck region. Furthermore, this proof-of-concept research established the feasibility of using recombinant Fab-dHLX constructs for in vivo imaging of tumor biomarkers.

\section{Introduction}

Antibody based molecular imaging provides a non-invasive means of detecting biological processes and molecular events in vivo. By combining the high sensitivity and resolution of e.g. a PET (positron emission tomography)-scanner with the tumor specificity of a tumor targeting molecule, this technique is of increasing importance to visualize and characterize tumor lesions. Additionally, it can be used for dosimetric calculations, to monitor therapy response and to identify patients who may benefit from a particular therapy (1). With advancements in instrumentation and development of novel targeted probes, immuno-PET has firmly established its role in drug development and in clinical assessment.

Although intact monoclonal antibodies (mAbs) have been considered candidates for molecular imaging due to their specificity and high signal delivery to cell surface molecules, their relatively large size $(\sim 150 \mathrm{kDa})$ and Fc-mediated half-life extension tend to cause suboptimal imaging pharmacokinetics, poorer tumor penetration and increased immunogenicity. Smaller targeting moieties such as antibody fragments have emerged as an alternative to mAbs to overcome the aforementioned limitations. Common antibody fragments are smaller molecules that omit the Fc-region and consist of one $\mathrm{Fab}(\sim 50 \mathrm{kDa})$ or two $\mathrm{F}\left(\mathrm{ab}^{\prime}\right)_{2}(100 \mathrm{kDa})$ antigen binding arms, respectively. Due to their smaller size, antibody fragments are likely to exhibit better extravasation and diffusion in the intracellular space and faster blood clearance compared to their 
parental antibodies (2). Consequently, normal tissue accumulation and immunologic reactions is expected to be lower with a fragment than with the intact antibody, thus enhancing tumor contrast and allowing imaging at earlier time-points. For several targets, antibody fragments have shown promising results as imaging agents in animals (3) and clinical studies (4). Generally, $F\left(a b^{\prime}\right)_{2}$ fragments are considered advantageous to Fab fragments in molecular imaging due to their bivalent nature, which can result in an increased functional affinity and tumor uptake compared to Fab. Another possible disadvantage of Fab fragments is that their molecular weight subjects them to glomerular filtration. The larger $F\left(a b^{\prime}\right)_{2}$ format, with a size above the renal threshold, is expected to exhibit a biodistribution shift from kidneys to liver (5).

An alternative to antibody fragments derived from full-length antibodies generated through immunization are recombinant Fabs obtained in vitro by phage display, generated from selections in phage libraries. Recombinant antibody technology is a rapidly evolving field with a number of major benefits over conventional antibody generation and production methods (6). It enables the use of in vitro selection steps that facilitate the isolation of antibodies with desired characteristics, e.g. antibodies that distinguish closely related antigens (7), and candidates can be engineered using readily available DNA sequences. Furthermore, since recombinant monoclonal antibody fragments can be produced in bacteria this allows for an easier, faster, and less expensive production process than using hybridoma or mammalian cell culture techniques. Moreover, antibodies selected from in vitro libraries of human antibody genes do not elicit the same immune responses in patients that are seen with non-human antibodies. Therefore, such antibodies can be used for therapeutic development. Thus, more and more fully human antibodies obtained from antibody libraries are entering clinical development and are reaching the market $(8)$.

We have developed the CD44v6-targeting human bivalent antibody fragment AbD19384, a recombinant human bivalent Fab antibody, engineered from monovalent Fab AbD15179 by subcloning of the Fab gene into an expression vector containing a self-dimerizing helix-turn helix motif (dHLX) $(9,10)$. The Fab-dHLX constructs represent a novel, attractive platform for cancer targeting. The size and format is functionally equivalent to a $\mathrm{F}\left(\mathrm{ab}^{\prime}\right)_{2}$ fragment, but there are also important differences besides the origin of the molecule. AbD19384 is a non-covalent homodimer formed via C-terminal fusion of a small homodimerization domain, whereas disulfide bridges are present in $F\left(a b^{\prime}\right)_{2}$ fragments enzymatically derived from full length antibodies. These non-covalent homodimers are stable under reducing conditions, whereas $F\left(a^{\prime}\right)_{2}$ fragments are not. CD44v6 was chosen as a target, since it is a surface antigen found to be overexpressed in $>90 \%$ of head and neck squamous cell carcinoma (HNSCC), as well as in other cancers such as lung, esophagus and breast, which makes it an attractive target for molecular imaging and targeted therapy (11). To the best of our knowledge, there are no previous studies of tumor targeting abilities of the Fab-dHLX format in vivo, hence it is not known how this construct functions in an in vivo setting. Thus, the present study is not only relevant for CD44v6-targeted molecular imaging, but also as the first study of a tumor targeting Fab-dHLX construct in vivo.
Consequently, the aim of the present study was to evaluate for the first time the in vitro and in vivo binding properties of the Fab-dHLX construct, and to assess the utility of radio-iodinated AbD19384 as a targeting agent for molecular imaging of CD44v6-expressing tumors.

\section{Materials and methods}

AbD19384. AbD19384 is a bivalent antibody fragment, engineered from two fully human AbD15179 Fab-fragments. This format is a non-covalent homodimer of AbD15179 formed via C-terminal fusion of a dHLX (synthetic double helix loop helix) dimerization motif $(9,10)$ to the heavy chain segment of AbD15179. This format, Fab-dHLX, also referred to as 'bivalent mini-antibody', has a molecular weight of $\sim 110 \mathrm{kDa}$, and is functionally equivalent to a F(ab') $)_{2}$ fragment. The dHLX helix-turn-helix motif is a de novo designed self-associating peptide $(9,12)$. The dimerization occurs spontaneously, and thus the construct is in equilibrium with the monomeric form and possibly also a small fraction of tetramers or higher order aggregates, with a majority of the molecules being in the dimeric form (9). The generation of the monovalent form AbD15179 has been previously described (7). AbD19384 was supplied by Bio-Rad AbD Serotec GmbH (Puchheim, Germany) in 3X PBS.

Surface plasmon resonance (SPR) analysis of AbD19384. Recombinant CD44v3-10 (7) and two BSA-conjugated peptides (Elim Biopharmaceuticals, Hayward, CA, USA) derived from human (CGNRWHEGYRQTPREDS) and murine (CQNGWQGKNPPTPSEDS) CD44v6, respectively, were immobilized by standard amine coupling on a ProteOn XPR36 general layer medium sensor chip (Bio-Rad Laboratories, Hercules, CA, USA). A v6-negative isoform of CD44 (CD44v3-10 $\Delta v 6$ ) was included as a negative control. Channels with immobilization levels of 200 RU (BSA-conjugated peptides), 500 RU (CD44v3-10) and 1000 RU (CD44v3-10 and CD44v3-10 $\Delta v 6$ ) were used. Dilution series (3-50 nM) of the Fab-fragment AbD15179 and its corresponding bivalent fragment AbD19384 in PBS 0.05\% Tween-20 (pH 7.4) were injected at $50 \mu \mathrm{l} / \mathrm{min}$ at $25^{\circ} \mathrm{C}$. Real-time signal from a blank channel and a parallel buffer injection were subtracted and binding data fitted to a 1:1 Langmuir isotherm using ProteOn Manager software version 3.1.0.6 (Bio-Rad Laboratories). Data were double referenced by subtraction of simultaneous responses from a blank surface and a buffer injection. Experimental data were plotted together with curves drawn from a fitted 1:1 Langmuir isotherm. Kinetic constants were calculated from five separate injections using two different immobilization levels of CD44v3-10. Surfaces were regenerated with $10 \mathrm{mM}$ $\mathrm{HCl}$ between injections.

Cell lines. The human SCC cell line A431 (obtained from the American Type Culture Collection, Manassas, VA, USA), derived from an epidermal carcinoma of the vulva, was cultured in Ham's F10, supplemented with $10 \%$ fetal calf serum, $2 \mathrm{mM}$ L-glutamine, and antibiotics (100 IU penicillin and $100 \mu \mathrm{g} / \mathrm{ml}$ streptomycin). A431 has been shown to be a highly CD44v6-expressing cell line with $\sim 3 \times 10^{6}$ antigens/ cell (13). The human SCC cell line H314 (obtained from the 
European Collection of Cell Cultures), derived from floor of the mouth, was cultured in a one-to-one mixture of Ham's F12 and Dulbecco's modified Eagle's medium (DMEM) with the same supplements. H314 has demonstrated a medium antigen density of $\sim 0.7 \times 10^{6} \mathrm{CD} 44 \mathrm{v} 6$ antigens per cell (14). The human SCC cell line UM-SCC74B derived from base of the tongue (kindly provided by Professor T.E. Carey, University of Michigan, MI, USA) was cultured in DMEM with the same supplements, as well as $1 \%$ non-essential amino acids. UM-SCC74B has demonstrated low expression $\left(\sim 0.1 \times 10^{5}\right)$ of CD44v6. As a negative control, the breast cancer cell line MDA-MB-231 (obtained from the American Type Culture Collection) was used. It was cultured in DMEM with the same supplements as described above for A431. This cell line has demonstrated no detectable CD44v6 expression (14). Cells were incubated at $37^{\circ} \mathrm{C}$ in an atmosphere containing $5 \% \mathrm{CO}_{2}$. For LigandTracer studies, $\sim 1 \times 10^{6}$ A 431 cells, $2 \times 10^{6} \mathrm{H} 314$ cells, $1 \times 10^{6}$ UM-SCC74B cells, or $2 \times 10^{6}$ MDA-MB-231 cells were seeded 2-3 days before measurements in a local part on a 10-cm cell dish (\#150350; Nunc) and allowed to adhere firmly to the plastics prior to the addition of fresh medium.

Labeling. AbD19384 was labeled with ${ }^{125}$ I (Perkin-Elmer, Waltham, MA, USA) using direct chloramine T labeling (CAT) (15) (Sigma-Aldrich). CAT and $\mathrm{Na}_{2} \mathrm{SO}_{5}$ (NBS) were dissolved in MilliQ-water to $4 \mathrm{mg} / \mathrm{ml}$. AbD19384 $(100 \mu \mathrm{g})$ in PBS was added to $10 \mathrm{MBq}$ of ${ }^{125} \mathrm{I}$ and mixed before adding $30 \mu \mathrm{l}$ of CAT. The reaction mixture was incubated for $1 \mathrm{~min}$ on ice before ending the reaction by adding $60 \mu \mathrm{l}$ of NBS. The sample was purified on a NAP5-size exclusion column (GE Healthcare Life Sciences Uppsala, Sweden) equilibrated with PBS.

Labeling of AbD19384 with ${ }^{124}$ I (Perkin-Elmer) using 1,3,4,6-Tetrachloro-3 $\alpha, 6 \alpha$-diphenylglycouril (Iodogen) was performed as following; three Iodogen buffers (A, B and C) were prepared; A, $0.5 \mathrm{M}$ sodium phosphate buffer, $\mathrm{pH} 7.4$; $\mathrm{B}$, $0.05 \mathrm{M}$ sodium phosphate and $5 \mathrm{M} \mathrm{NaCl}, \mathrm{pH} 7.4 ; \mathrm{C}, 0.05 \mathrm{M}$ sodium phosphate, $5 \%$ potassium iodide and $0.5 \% \mathrm{BSA}(\mathrm{w} / \mathrm{v})$. All buffers were prepared using MilliQ-water. Iodogen was dissolved in dichloromethane to $0.2 \mathrm{mg} / \mathrm{ml} .{ }^{124} \mathrm{I}$ was incubated with cold $\mathrm{NaI}$ in a 1:1 molar ratio with the added AbD19384 in order to ensure that the nuclide was in iodide form prior to starting the labeling procedure. ${ }^{124} \mathrm{I}$ and AbD19384 $(1 \mathrm{mg} / \mathrm{ml}$ in PBS) were mixed in a tube previously coated with $50 \mu \mathrm{g}$ Iodogen. Buffer A was added in an equivalent volume, incubated at room temperature for $7 \mathrm{~min}$ and agitated carefully every $30 \mathrm{sec}$ using a vortex. The reaction mixture was transferred to a new tube and buffer B (480 $\mu \mathrm{l})$ was subsequently added. Following at least 10 min of rest, buffer C (480 $\mu \mathrm{l})$ was added, and the sample was mixed thoroughly. Labeled $\mathrm{F}\left(\mathrm{ab} \mathrm{b}_{2}\right.$ was separated from non-reacted radionuclide and low-molecular-weight reaction components by using a NAP-5 or PD10 column pre-equilibrated with PBS. Iodination using Iodogen was also performed using ${ }^{125} \mathrm{I}$ in the same way, in order to optimize and compare labelings.

To determine the yield, purity and stability of the labeled conjugates, instant thin-layer chromatography (ITLC) analyses were performed on labeled conjugates. Samples taken immediately as well as $48 \mathrm{~h}$ after the labeling procedure were analyzed. Serum stability tests were performed by $1 \mathrm{~h}$ incubation at $37^{\circ} \mathrm{C}$ in $42 \%$ murine serum in PBS, $\mathrm{pH} 7.4$. Approximately $1 \mu \mathrm{l}$ of the conjugate was placed on an ITLC chromatography strip (Biodex) and placed into a 'running buffer' (70\% acetone), followed by measurements on a Cyclone Storage Phosphor system. Data were analyzed using OptiQuant image analysis software.

In vitro binding measurements on cultured tumor cells. Real-time in vitro binding and retention measurements of the radiolabeled conjugates were performed at room temperature using LigandTracer instruments (Ridgeview Instruments AB, Uppsala, Sweden) on CD44v6-positive A431, H314 and UM-SCC74B cells. In vitro binding specificity measurements were performed on LigandTracer instruments using MDA-MB-231 cells as negative controls. LigandTracer Grey was used for ${ }^{125} \mathrm{I}-\mathrm{AbD} 19384$ and LigandTracer White was used for ${ }^{124} \mathrm{I}-\mathrm{AbD} 19384$. Binding traces using several subsequent concentrations $(0-90 \mathrm{nM})$ were obtained for at least $1 \mathrm{~h}$ per concentration, followed by a dissociation measurement for at least $15 \mathrm{~h}$. The shapes of the real-time binding curves produced in LigandTracer were compared in the evaluation software TraceDrawer 1.6.1 (Ridgeview Instruments AB, Vänge, Sweden). Through kinetic fitting using a 1:1 and 1:2 models, the dissociation equilibrium constant $\mathrm{K}_{\mathrm{D}}$ (corresponding to the apparent affinity), the association rate constant $\mathrm{k}_{\mathrm{a}}$ and the dissociation rate $\mathrm{k}_{\mathrm{d}}$ were obtained.

Small animal studies. Female nu/nu Balb/c mice were housed under standard laboratory conditions and fed ad libitum. All experiments complied with the Swedish law and were performed with permission from the Uppsala Committee of Animal Research Ethics, ethical permission number C 410/12.

In vivo specificity of ${ }^{125} I-A b D 19384$. In the first group $(\mathrm{n}=5)$, aimed at analyzing in vivo specific binding of ${ }^{125} \mathrm{I}-\mathrm{AbD} 19384$ to CD44v6, approximately $\left(8 \times 10^{6}\right)$ A431 cells (high CD44v6 expression) suspended in $150 \mu 1$ 1:1 cell medium:Matrigel were injected s.c. into the right posterior leg. In the same mice, $\sim 8 \times 10^{6}$ MDA-MB-231 cells (no CD44v6 expression) suspended in $150 \mu 1$ 1:1 cell medium:Matrigel were injected in the left posterior leg. Two weeks after tumor cell injections, experiments were performed. At the time of study, average animal weights were $19.9 \pm 0.9 \mathrm{~g}$ (SEM), A431 tumor weights $73 \pm 20 \mathrm{mg}$, and MDA-MB-231 tumor weights $169 \pm 23 \mathrm{mg}$. The mice were injected with ${ }^{125} \mathrm{I}-\mathrm{AbD} 19384(11 \mu \mathrm{g}$ in $200 \mu \mathrm{l}$ PBS, $100 \mathrm{kBq}$ ) in the tail vein. At $24 \mathrm{~h}$ post injection (p.i), animals were euthanized with a mixture of ketamine and xylazine followed by heart puncture. Blood and tumors were collected and weighed, and tracer uptake was measured in a gamma well-counter (1480 Wizard; Wallace Oy, Turku, Finland). Radioactivity uptake in the organ was calculated as percent of injected activity per gram of tissue $(\% \mathrm{ID} / \mathrm{g})$. Tumor-to-blood ratio was calculated as activity $/ \mathrm{g}_{\text {tumor }}$ divided by activity/g $\mathrm{g}_{\text {blood }}$.

Ex vivo biodistribution of ${ }^{125} I-A b D 19384$. In a second group $(\mathrm{n}=20)$, used to analyze in vivo binding of ${ }^{125} \mathrm{I}-\mathrm{AbD} 19384$ to xenografts with varying CD44v6 receptor densities, approximately $\left(8 \times 10^{6}\right)$ A431 cells suspended in $150 \mu 1$ 1:1 cell medium:Matrigel were injected s.c. into the left posterior 
leg and $10 \times 10^{6} \mathrm{H} 314$ cells (moderate CD44v6 expression) suspended in $150 \mu 1$ 1:1 cell medium:Matrigel were injected in the right posterior leg. Three weeks after tumor cell injections, experiments were performed. At the time of study, average animal weights were $20.1 \pm 1.3 \mathrm{~g}$, A431 tumor weights $219 \pm 54 \mathrm{mg}$ and H314 tumor weights $23 \pm 3 \mathrm{mg}$. Twenty mice bearing dual A431 (high CD44v6 expression) and H314 (moderate CD44v6 expression) xenografts received an intravenous injection via the tail vein with ${ }^{125} \mathrm{I}-\mathrm{AbD} 19384(100 \mathrm{kBq})$, totally $11 \mu \mathrm{g} \mathrm{F}\left(\mathrm{ab}^{\prime}\right)_{2}$ in $200 \mu \mathrm{l}$ PBS per mouse. Animals were sub-divided into four groups with five mice in each. At 6, 24, 48 and 72 h p.i. animals were euthanized with a mixture of ketamine and xylazine followed by heart puncture. Blood was collected, and tumors and organs of interest such as salivary glands, thyroid (en bloc with larynx), tongue, heart, liver, kidneys, spleen, urinary bladder, colon, upper gastrointestinal tract, skin, bone and muscle were excised, weighed and measured in a gamma well-counter. The tail and the rest of the body were also measured. Three injection standards were measured for each group. Radioactivity uptake in the organ was calculated as percent of injected activity per gram of tissue (\%ID/g). Thyroid was excised en bloc with larynx, and uptake was calculated as percent of injected activity per organ. Tumor to organ ratio was calculated as activity $/ \mathrm{g}_{\text {tumor }}$ divided by activity/gorgan .

In vivo PET/CT and ex vivo biodistribution studies with ${ }^{124} I-A b D 19384$. A third group was used to analyze in vivo biodistribution of ${ }^{124} \mathrm{I}-\mathrm{AbD} 19384(\mathrm{n}=12)$ or ${ }^{18} \mathrm{~F}-\mathrm{FDG}(\mathrm{n}=4)$ in mice with xenografts with varying $\mathrm{CD} 44 \mathrm{v} 6$ receptor densities and for PET/CT imaging. Approximately $\left(8 \times 10^{6}\right)$ A431 cells suspended in $150 \mu 1$ 1:1 cell medium:Matrigel were injected s.c. into the left posterior leg and $10 \times 10^{6} \mathrm{UM}-\mathrm{SCC} 74 \mathrm{~B}$ tumor cells (low CD44v6 expression) suspended in 150 $\mu 1$ 1:1 cell medium:Matrigel were injected in the right posterior leg. Tumors were allowed to grow for three weeks before start of experiments. At the time of study, average animal weights were $16.1 \pm 1.5 \mathrm{~g}$. A day before conjugate injections, mice were given potassium iodide $(1 \%)$ in drinking water to block uptake of free ${ }^{124} \mathrm{I}$ in thyroid. Animals were then injected with ${ }^{124} \mathrm{I}-\mathrm{AbD} 19384(11 \mu \mathrm{g}, 1.5 \mathrm{MBq}$, in $200 \mu \mathrm{l}$ PBS per mouse) in the tail vein as a single bolus. As verified with native gel analysis, $17 \%$ of injected ${ }^{124} \mathrm{I}-\mathrm{AbD} 19384$ consisted of monomers or smaller fragments.

Whole body PET/CT studies of ${ }^{124} \mathrm{I}-\mathrm{AbD} 19384$ were performed under general anesthesia (isoflurane 1.0-2.5\% in $50 \% / 50 \%$ medical oxygen:air at $450 \mathrm{ml} / \mathrm{min}$ ) at 24,48 and $72 \mathrm{~h}$ p.i. Four additional mice were imaged using ${ }^{18} \mathrm{~F}-\mathrm{FDG}$ (2.7 Mbq) at $30 \mathrm{~min}$ p.i. under general anesthesia. Sedated xenograft animals with pre-injected tracer were placed in the gantry of the small animal PET/CT scanner Triumph $^{\mathrm{TM}}$ Trimodality System; TriFoil Imaging, Inc., Northridge, CA, USA). Whole body PET scans were performed for $80 \mathrm{~min}$ in list mode followed by a CT examination for $3 \mathrm{~min}$ (Field of View $=8.0 \mathrm{~cm}) .{ }^{18} \mathrm{~F}$-FDG PET scan was acquired for $60 \mathrm{~min}$. At indicated times, animals were euthanized, and organs removed and measured as described above. The breathing rate was monitored with a camera under controlled anesthesia. Animals were placed on the heated bed of the PET scanner to prevent hypothermia and fastened to prevent large movements during study. ${ }^{18}$ F-FDG was kindly provided by the Nuclear Medicine Department (Akademiska Sjukhuset, Uppsala, Sweden).

The PET data were reconstructed into a static image using an ordered subset expectation maximization 3-D algorithm (20 iterations). The CT raw files were reconstructed using Filter Back Projection. PET data were reconstructed for attenuation and scatter corrections with their respective CT data. PET and CT dicom files were analyzed using PMOD v3.508 (PMOD Technologies Ltd., Zurich, Switzerland). Volumes of interest were drawn manually on tumors, muscle and left ventricle. The measurement from left ventricle was accounted for blood. Tracer uptake in these organs from PET images is expressed as tissue-to-reference tissue ratio. Blood was used as reference tissue.

After PET/CT measurements, animals were euthanized; blood, tumors and organs of interest were collected and treated as described above for ex vivo biodistribution measurements. For animals injected with ${ }^{124} \mathrm{I}-\mathrm{AbD} 19384(\mathrm{n}=12)$, A431 tumor weights were $567 \pm 61 \mathrm{mg}$, and UM-SCC74B tumor weights $483 \pm 77 \mathrm{mg}$. For animals injected with ${ }^{18} \mathrm{~F}-\mathrm{FDG}(\mathrm{n}=4) \mathrm{A} 431$ tumor weights were $681 \pm 210 \mathrm{mg}$, and UM-SCC74B tumor weights $409 \pm 241 \mathrm{mg}$ at the time of experiments.

Statistical analyses. Statistical analyses were performed using GraphPad Prism version 5.02 for Windows (GraphPad Software Inc., La Jolla, CA, USA). For in vivo studies, the differences in uptake between MDA-MB-231/UM-SCC74B tumors and A431 tumors were assessed using a two-tailed $\mathrm{t}$-test and were considered statistically significant at $\mathrm{P}<0.05$. The biodistribution data are presented as mean \pm standard deviation (SD). Significant differences between the groups over time were tested with one-way analysis of variance (ANOVA) with Newman-Keuls multiple comparison test. The differences were considered statistically significant at $\mathrm{P}<0.05$. The differences in uptake of ${ }^{125} \mathrm{I}-\mathrm{AbD} 19384$ and ${ }^{124} \mathrm{I}-\mathrm{AbD} 19384$ between low/medium CD44v6 expressing tumors and high CD44v6 expressing tumors were evaluated using repeated measures ANOVA with Newman-Keuls multiple comparison test and were considered statistically significant at $\mathrm{P}<0.05$.

\section{Results}

SPR analysis. Both AbD19384 and AbD15179 bound CD44v3-10, representative sensorgrams are shown in Fig. 1, and no binding was seen to the negative control isoform. Reformatting Fab AbD15179 into the bivalent AbD19384 improved the apparent affinity $\left(\mathrm{K}_{\mathrm{D}}\right)$ almost 6 -fold from 6 to $1 \mathrm{nM}$. This was a result of an improved dissociation rate, which was decreased from $1.1( \pm 0.3) \times 10^{-3} \mathrm{~s}^{-1}$ for AbD15179 to $1.6( \pm 0.5) \times 10^{-4} \mathrm{~s}^{-1}$ for AbD19384. The association rate constants were similar, $1.8( \pm 0.5) \times 10^{5} \mathrm{M}^{-1} \mathrm{~s}^{-1}$ for $\mathrm{AbD} 15179$ vs. $1.4( \pm 0.8)$ $\mathrm{x} 10^{5} \mathrm{M}-{ }^{1} \mathrm{~s}^{-1}$ for the larger AbD19384. Both antibody fragments bound human CD44v6 peptide and no signals were observed on surfaces immobilized with murine CD44v6 peptide (data not shown).

Labeling. Labeling yields were $64-74 \%$ for ${ }^{125} \mathrm{I}-\mathrm{AbD} 19384$, and $64-73 \%$ for ${ }^{124} \mathrm{I}-\mathrm{AbD} 19384$, respectively. Specific activity for ${ }^{125} \mathrm{I}-\mathrm{AbD} 19384$ was adjusted before in vivo injections, using unlabeled AbD19384, resulting in an injected activity dose 

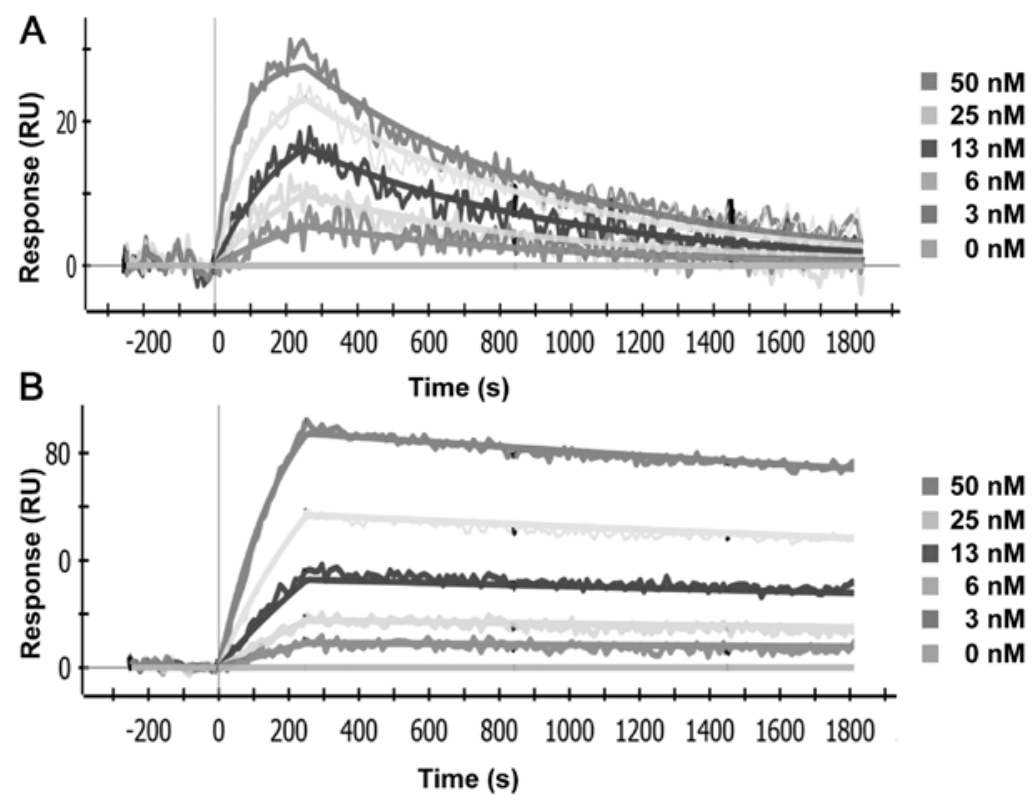

Figure 1. Representative data from SPR analyses of fragments binding to CD44v3-10. Five concentrations of each fragment ranging from 3 to 50 nM were injected over a surface with immobilized CD44v3-10 on a ProteOn XPR36 protein interaction array system (Bio-Rad). (A) AbD15179 and (B) AbD19384.

of $100 \mathrm{kBq}$ per mouse (11 $\mu \mathrm{g}$ AbD19384 per mouse). The specific activity of the injection solution for ${ }^{124} \mathrm{I}-\mathrm{AbD} 19384$ was $136 \mathrm{kBq} / \mu \mathrm{g}$, resulting in an injected activity dose of $1.5 \mathrm{MBq}(11 \mu \mathrm{g})$ per mouse. The radiochemical purity after size-exclusion chromatography purification was $>95 \%$ for all conjugates. Radiochemical purity of labeled conjugates stored in PBS for $48 \mathrm{~h}$, or in serum for $1 \mathrm{~h}$, was unchanged according to ITLC analysis for both conjugates. Native gel analyses of radioiodinated conjugates demonstrated that serum incubation did not influence either monomer/dimer equilibrium, or aggregation of the conjugate (data not shown).

In vitro binding measurements of iodinated AbD19384 on cultured tumor cells. Real-time binding data from measurements of radio-iodinated AbD19384 binding to cells can be seen in Fig. 2. A clear binding with slow off-rate was seen on both A431 and H314 cells, whereas a lower, noisier signal was seen for the low CD44v6-expressing UM-SCC74B cells and no signal was detected on the negative control MDA-MB-231 cells (Fig. 2A). All iodinated conjugates behaved in a similar manner with comparable binding and retention for conjugates regardless of labeling method (using CAT or Iodogen) or nuclide $\left({ }^{125} \mathrm{I}\right.$ or $\left.{ }^{124} \mathrm{I}\right)$ (Fig. 2B). Interactions were mainly $1: 1$ and the apparent $\mathrm{K}_{\mathrm{D}}$ of the high-affinity interaction was around $1 \mathrm{nM}$ for all three conjugates $(0.8 \pm 0.1,0.7 \pm 0.12$ and $1.3 \pm 0.2 \mathrm{nM}$ for ${ }^{125} \mathrm{I}-\mathrm{AbD} 19384$ (CAT), ${ }^{125} \mathrm{I}-\mathrm{AbD} 19384$ (Iodogen), and ${ }^{124} \mathrm{I}-$ AbD19384 (Iodogen), respectively).

\section{Small animal studies}

In vivo specificity of AbD19384. Tumor uptake in A431 tumors was $0.97 \pm 0.17 \% \mathrm{ID} / \mathrm{g}$, and uptake in MDA-MB-231tumors was $0.24 \pm 0.04 \% \mathrm{ID} / \mathrm{g}$, comparable to amounts in blood $(0.23 \pm 0.11 \% \mathrm{ID} / \mathrm{g})$. The difference in uptake was statistically significant $(\mathrm{P}<0.0001)$, with the CD44v6 positive tumors displaying approximately four times higher uptake compared to the CD44v6 negative tumors.
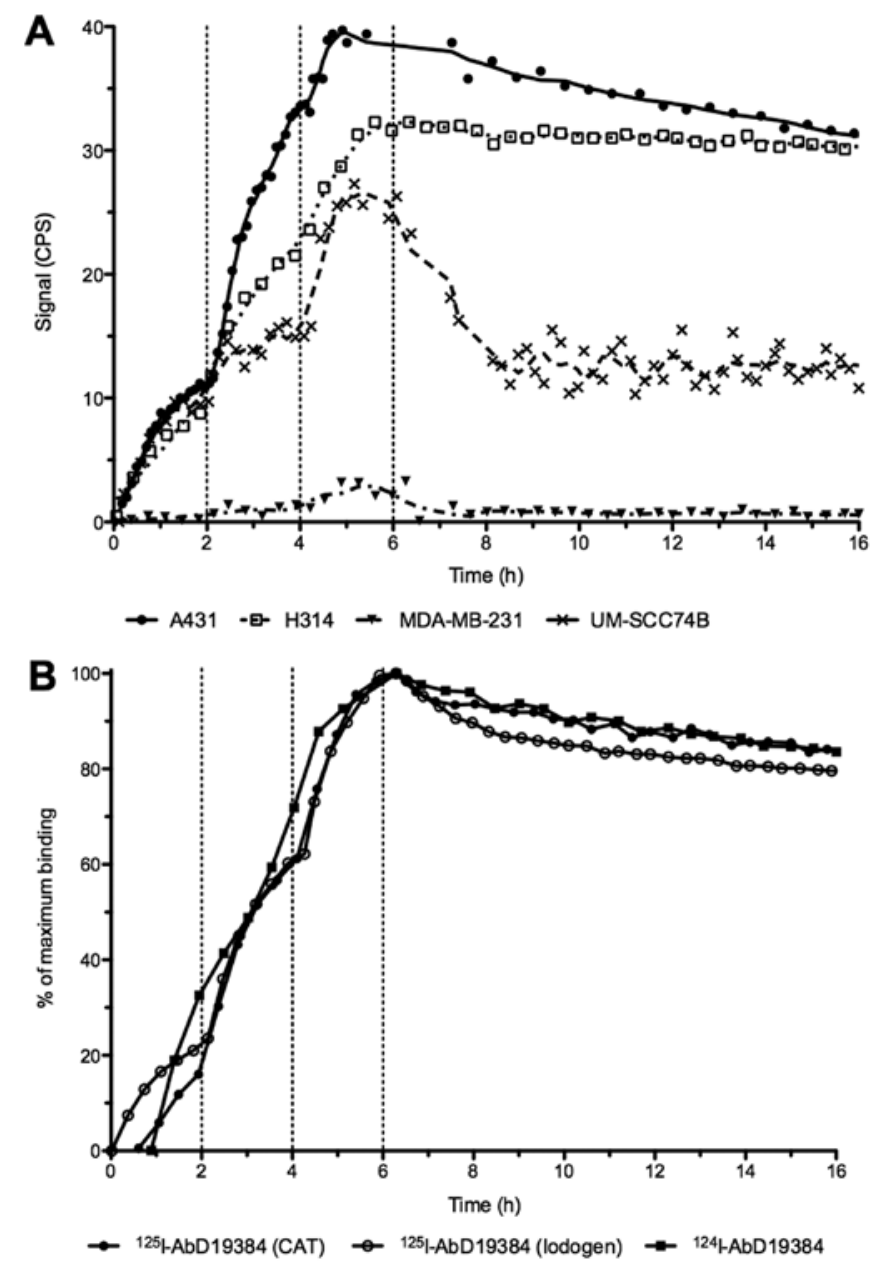

Figure 2. Representative LigandTracer measurements of real-time binding Binding traces using three subsequent concentrations $(10,30$ and $90 \mathrm{nM})$ were obtained for at least $1 \mathrm{~h}$ per concentration (marked by vertical dotted lines), followed by a dissociation measurement for at least $15 \mathrm{~h}$. (A) Binding of ${ }^{125} \mathrm{I}$-AbD19384 to cell lines of various CD44v6 density. Signal is shown as counts per second (CPS). (B) Comparison of iodinated AbD19384 conjugates on A431 cells. Curves were normalized to percentage of maximum binding. 

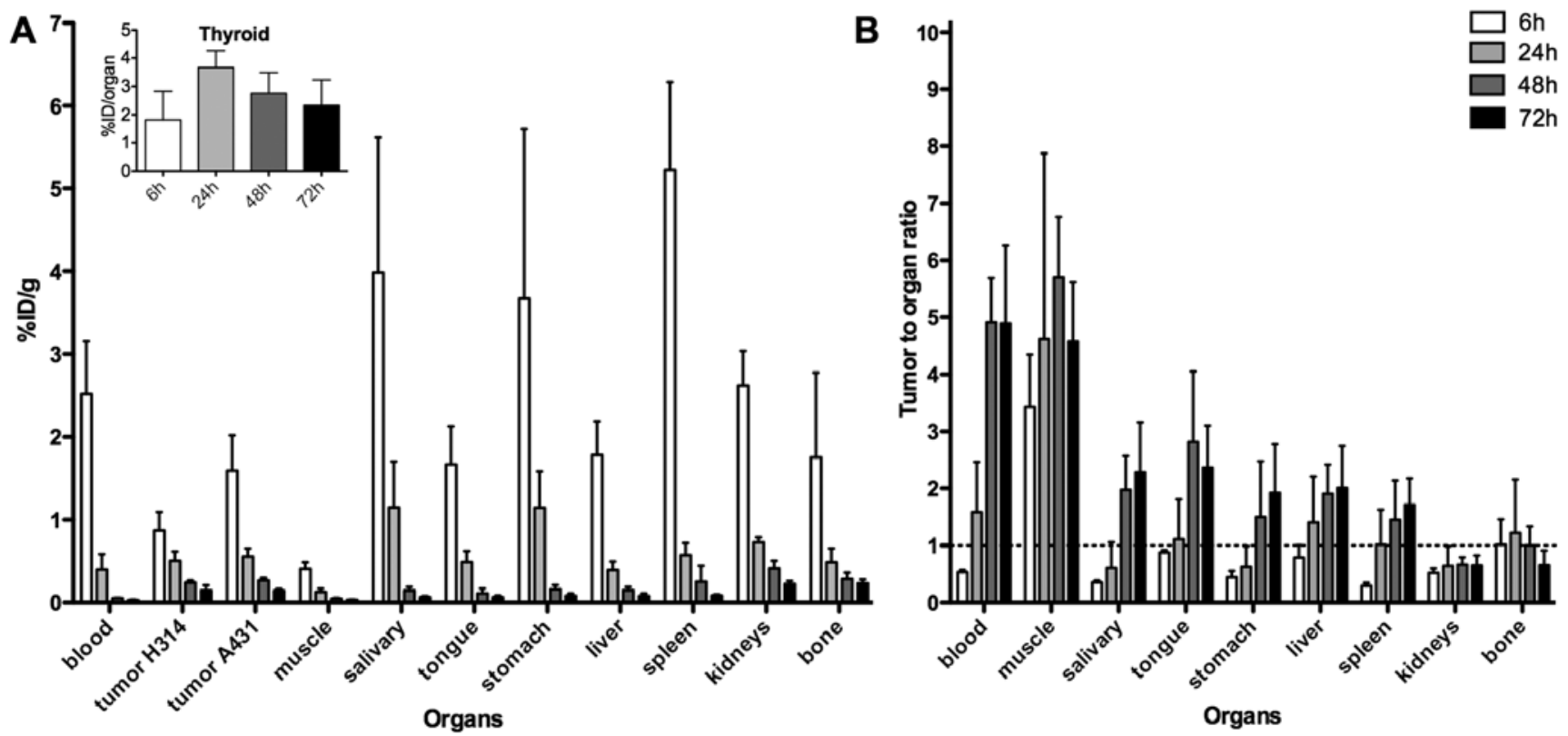

Figure 3. (A) Biodistribution and (B) A431 tumor-to-organ ratios of ${ }^{125} \mathrm{I}-\mathrm{AbD} 19384$ in tumors and selected organs of tumor-bearing mice. Inset shows $\% \mathrm{ID} /$ organ for thyroid. Data are expressed as percentage of injected activity per gram of tissue (\%ID/g). Error bars, SD (N=5).
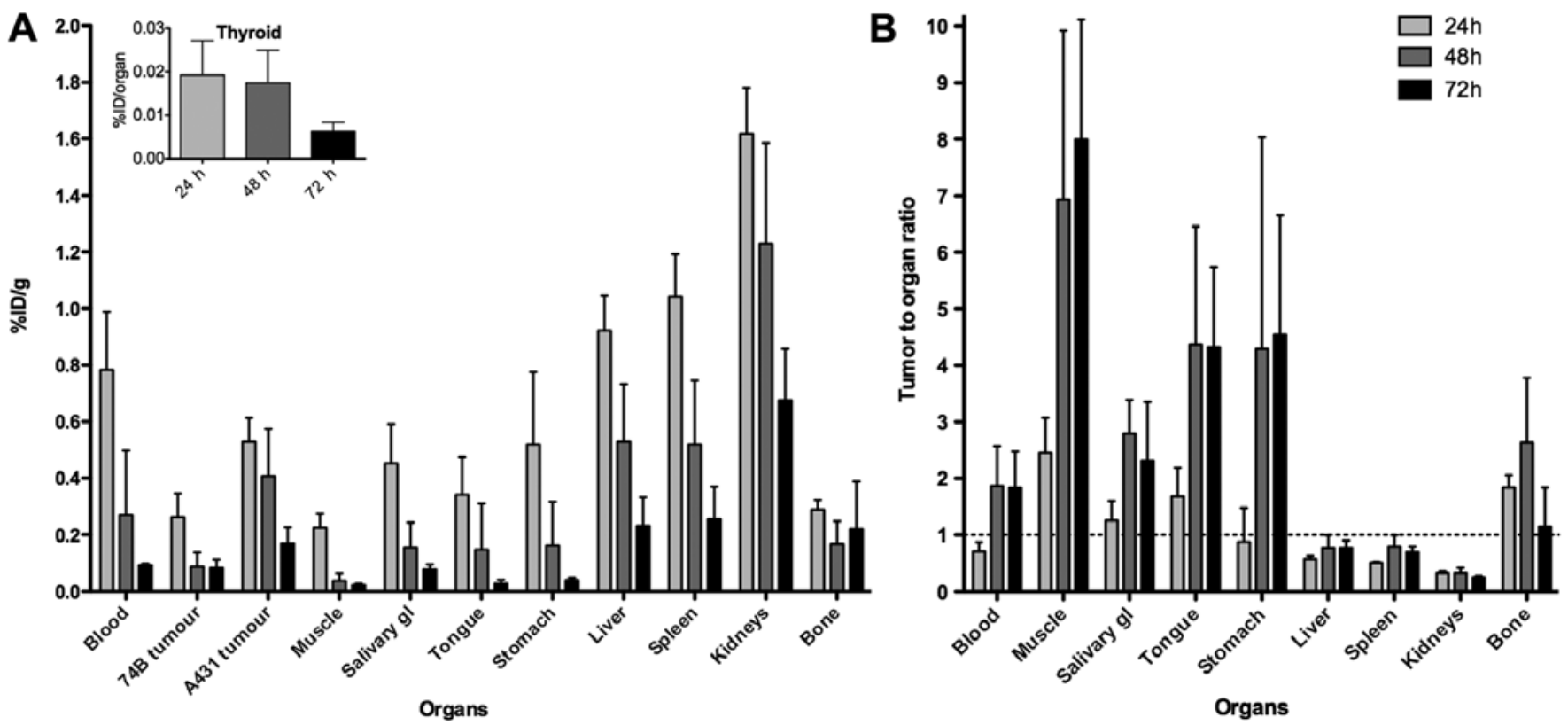

Figure 4. (A) Biodistribution and (B) A431 tumor-to-organ ratios of ${ }^{124} \mathrm{I}-\mathrm{AbD} 19384$ in tumors and selected organs of tumor-bearing mice. Inset shows \%ID/ organ for thyroid. Data are expressed as percentage of injected activity per gram of tissue (\%ID/g). Error bars, SD (N=4).

Ex vivo biodistribution study using ${ }^{125}$ I-AbD19384. Uptake of ${ }^{125} \mathrm{I}-\mathrm{AbD} 19384$ in tumors, blood, and selected organs can be seen in Fig. 3A, and a comparison between ${ }^{125} \mathrm{I}-\mathrm{AbD} 19384$ and ${ }^{125} \mathrm{I}-\mathrm{AbD} 15179$ uptake in liver and spleen is shown in Table I. A pronounced clearance and decrease in activity over time was seen for all organs, except thyroid, where activity remained stable over time (inset, Fig 3A). At $6 \mathrm{~h}$ p.i., a significant difference $(\mathrm{P}=0.02)$ in tumor uptake between the high- and moderate-CD44v6-expressing tumors was observed, with $1.60 \pm 0.42 \% \mathrm{ID} / \mathrm{g}$ in the high expressing A431 tumor, compared to $0.92 \pm 0.24 \% \mathrm{ID} / \mathrm{g}$ in the low expressing
H314 tumor. This difference did, however, disappear over time. Activity in blood at $6 \mathrm{~h}$ p.i. was $2.52 \pm 0.64 \% \mathrm{ID} / \mathrm{g}$, declining to $0.33 \pm 0.22 \% \mathrm{ID} / \mathrm{g}$ at $24 \mathrm{~h}$ p.i. and $0.03 \pm 0.01 \% \mathrm{ID} / \mathrm{g}$ at the latest time-point. The organs with the highest initial activity were the spleen, the stomach and the salivary glands. Tumor-toorgan-ratios, calculated based on the A431 tumors, can be seen in Fig. 3B. Tumor-to-organ ratios for ${ }^{125} \mathrm{I}-\mathrm{AbD} 19384$ increased over time for most organs of interest, as well as for blood up to $48 \mathrm{~h}$ p.i. Tumor to blood ratio for ${ }^{125} \mathrm{I}-\mathrm{AbD} 19384$ was below one at $6 \mathrm{~h}$ p.i., however, at $72 \mathrm{~h}$ the ratio had increased to $4.90 \pm 1.38 \% \mathrm{ID} / \mathrm{g}$. Tumor-to-organ ratios above 
Table I. Comparison of \%ID/g of ${ }^{125} \mathrm{I}-\mathrm{AbD} 19384$ and ${ }^{125} \mathrm{I}-\mathrm{AbD} 15179$ (18) in liver and kidneys, as obtained by ex vivo organ distribution.

\begin{tabular}{|c|c|c|c|c|c|c|c|c|c|}
\hline \multirow[b]{2}{*}{ Organ } & \multirow[b]{2}{*}{ Tracer } & \multicolumn{2}{|c|}{$6 \mathrm{~h}$} & \multicolumn{2}{|c|}{$24 \mathrm{~h}$} & \multicolumn{2}{|c|}{$48 \mathrm{~h}$} & \multicolumn{2}{|c|}{$72 \mathrm{~h}$} \\
\hline & & Mean & SD & Mean & SD & Mean & SD & Mean & SD \\
\hline \multirow[t]{2}{*}{ Blood } & AbD15179 & 1.97 & 0.35 & 0.39 & 0.08 & 0.14 & 0.11 & 0.051 & 0.008 \\
\hline & AbD19384 & 2.52 & 0.64 & 0.40 & 0.19 & 0.054 & 0.003 & 0.030 & 0.006 \\
\hline \multirow{2}{*}{$\begin{array}{l}\text { Tumor } \\
\text { (A431) }\end{array}$} & AbD15179 & 1.58 & 0.12 & 0.60 & 0.09 & 0.44 & 0.27 & 0.22 & 0.04 \\
\hline & AbD19384 & 1.59 & 0.43 & 0.56 & 0.10 & 0.27 & 0.03 & 0.14 & 0.02 \\
\hline \multirow[t]{2}{*}{ Liver } & AbD15179 & 1.09 & 0.19 & 0.30 & 0.06 & 0.12 & 0.06 & 0.067 & 0.012 \\
\hline & AbD19384 & 1.78 & 0.41 & 0.39 & 0.10 & 0.15 & 0.04 & 0.078 & 0.032 \\
\hline \multirow[t]{2}{*}{ Kidneys } & AbD15179 & 6.46 & 2.18 & 1.37 & 0.25 & 0.78 & 0.31 & 0.57 & 0.09 \\
\hline & AbD19384 & 2.62 & 0.42 & 0.73 & 0.06 & 0.41 & 0.09 & 0.22 & 0.04 \\
\hline
\end{tabular}

Six, 24, 48 and $72 \mathrm{~h}$ refer to time post-administration of tracer. $(\mathrm{N}=5)$.

Table II. Comparison of tumor uptake of ${ }^{124} \mathrm{I}-\mathrm{AbD} 19384$ relative to blood (heart), as obtained by PET imaging and ex vivo organ distribution.

\begin{tabular}{|c|c|c|c|c|c|c|c|c|c|c|}
\hline \multirow[b]{2}{*}{ Tumor type } & \multirow[b]{2}{*}{ Mode } & \multicolumn{3}{|c|}{$24 \mathrm{~h}$} & \multicolumn{3}{|c|}{$48 \mathrm{~h}$} & \multicolumn{3}{|c|}{$72 \mathrm{~h}$} \\
\hline & & Mean & SD & $\mathrm{N}$ & Mean & $\mathrm{SD}$ & $\mathrm{N}$ & Mean & $\mathrm{SD}$ & $\mathrm{N}$ \\
\hline \multirow[t]{2}{*}{ A431 } & $P E T$ & 0.83 & 0.18 & 4 & 1.32 & 0.32 & 3 & 1.44 & 0.58 & 3 \\
\hline & Ex vivo & 0.71 & 0.17 & 4 & 1.86 & 0.71 & 4 & 1.83 & 0.64 & 4 \\
\hline \multirow[t]{2}{*}{ UM-SCC74B } & PET & 0.33 & 0.03 & 3 & 0.49 & 0.36 & 3 & 0.99 & 0.29 & 3 \\
\hline & Ex vivo & 0.34 & 0.04 & 4 & 0.52 & 0.38 & 4 & 0.90 & 0.29 & 4 \\
\hline
\end{tabular}

Twenty-four, 48 and $72 \mathrm{~h}$ refer to time post-administration of ${ }^{124} \mathrm{I}-\mathrm{AbD} 19384$.

one were demonstrated in the organs in the head and neck region at the later time-points.

Ex vivo biodistribution and in vivo small animal PET study with ${ }^{124} \mathrm{I}-A b D 19384$. Uptake of ${ }^{124} \mathrm{I}-\mathrm{AbD} 19384$ in tumors, blood, and selected organs, as assessed by ex vivo organ distribution, are presented in Fig. 4A. A pronounced clearance and decrease in activity over time was seen for all organs, except thyroid, where activity was very low, and remained stable over time (inset, Fig 4A). At all three time-points, a significant difference in tumor uptake between the high- and low-CD44v6-expressing tumors was observed, with A431 tumors displaying on average 2.3, 3.5 and 2.0 times higher uptake than UM-SCC74B tumors at 24, 48 and $72 \mathrm{~h}$ p.i., respectively. Activity in blood at $24 \mathrm{~h}$ p.i. was $0.78 \pm 0.21 \% \mathrm{ID} / \mathrm{g}$, declining to $0.09 \pm 0.005 \% \mathrm{ID} / \mathrm{g}$ at $72 \mathrm{~h}$ p.i. The organs with the highest initial activity were the kidneys, the spleen and the liver. Tumor-to-organ ratios, calculated based on the A431 tumors, can be seen in Fig. 4B, and tumor-to-blood ratios are listed in Table II. Tumor-to-organ ratios were below one for liver, spleen and kidneys at all time-points, and above two for the late time-points in the organs in the head and neck region.
Biodistribution data verified that animals imaged by PET were representative for each group.

Representative PET images of ${ }^{124} \mathrm{I}-\mathrm{AbD} 19384$ at 24, 48 and $72 \mathrm{~h}$ p.i., as well as ${ }^{18}$ F-FDG 30 min p.i., can be seen in Fig. 5. The high CD44v6-expressing A431 tumors were visualized at all three time-points, with $48 \mathrm{~h}$ p.i. providing best contrast. UM-SCC74B tumors were visualized to a lower extent, in line with the biodistribution data of the conjugate. Tumor to blood ratios were also calculated from the PET examinations and compared to ratios generated from ex vivo biodistribution measurements (Table I). Results were in good agreement (no statistical differences between any pair of measurements). ${ }^{18}$ F-FDG demonstrated faint visualization of tumors, no discrepancy between the high- and low-CD44v6 expressing tumor, and some uptake in brown adipose tissue (Fig. 5).

\section{Discussion}

The objective of the present study was to evaluate for the first time the in vitro and in vivo binding properties of the radioiodinated human bivalent fragment AbD19384 for targeting of CD44v6-expressing tumors, and to assess the utility of 


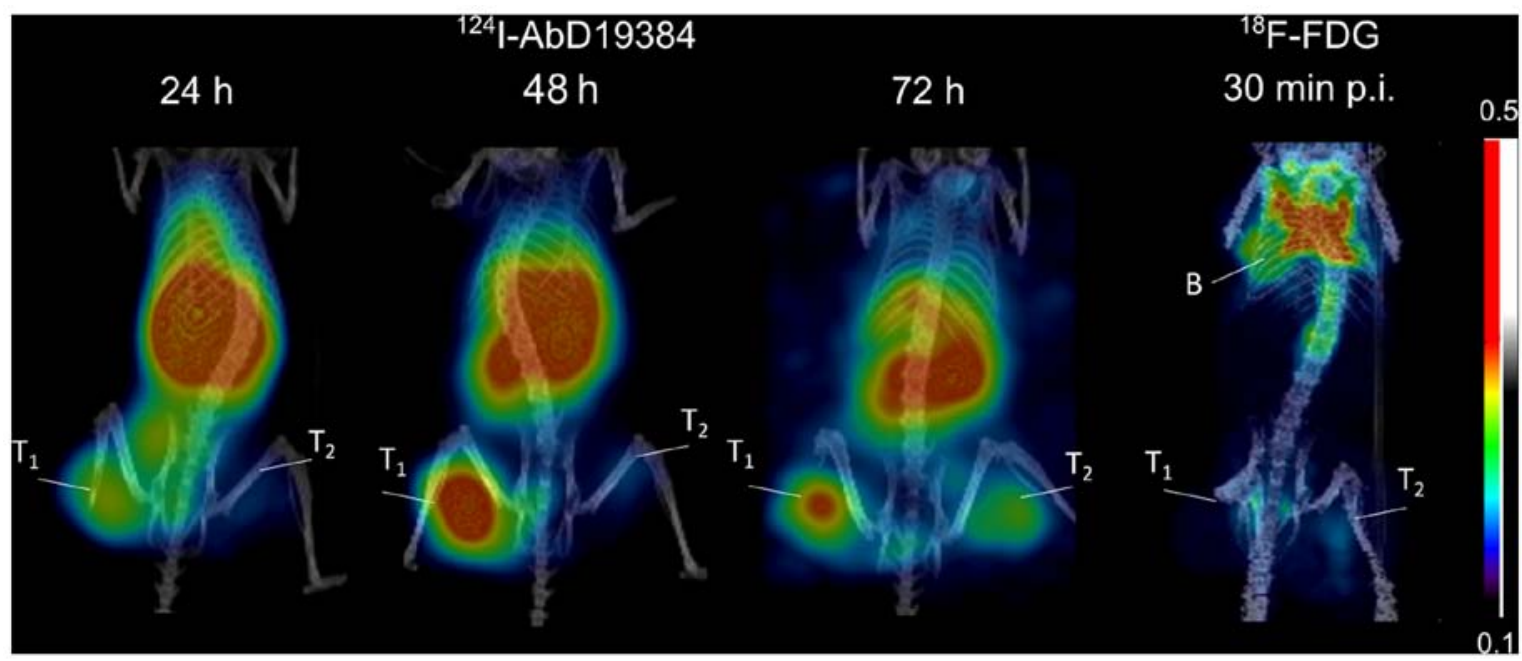

Figure 5. Small animal PET imaging of ${ }^{124} \mathrm{I}$ in mice bearing high CD44v6-expressing tumors on the left ' $\mathrm{T} 1$ ' and low CD44v6-expressing tumors on the right 'T2'. Uptake is also seen in kidneys, liver and spleen. ${ }^{18} \mathrm{~F}$-FDG-PET is shown on the right at $30 \mathrm{~min}$ p.i., showing uptake in brown fat ' $\mathrm{B}$ '.

Fab-dHLX constructs as targeting agents for molecular imaging. As CD44v6 is overexpressed in a number of epithelial tumors AbD19384 could prove to be valuable as a targeting molecule for a number of cancers, for example HNSCC. To the best of our knowledge, this is the first time a Fab-dHLX construct has been used for in vivo targeting and molecular imaging. Previous in vivo studies using a dHLX construct have, however, assessed the possibility of antibody-mediated toxicity neutralization in mice using Fab-dHLX (16), or scFvdHLX (17) by pre-incubation of targeting molecule and target with promising results.

In the present study, antigen specificity, binding properties, and interaction analysis of AbD19384 were first assessed in vitro. In vivo specificity and biodistribution of ${ }^{125} \mathrm{I}-\mathrm{AbD} 19384$ was then evaluated in tumor-bearing mice using a dual-tumor setup. Finally, AbD19384 was labeled with ${ }^{124}$ I, and its imaging properties were assessed in mice bearing tumors of both low and high CD44v6 expression using small animal PET/CT and compared to ${ }^{18} \mathrm{~F}-\mathrm{FDG}$.

SPR measurements clearly demonstrated in vitro specificity of AbD19384, and an improved apparent affinity compared to the monovalent AbD15179 fragment (Fig. 1). AbD19384 did not bind to the murine v6-peptide. Binding of iodinated AbD19384 conjugates was also followed in real-time in vitro on cultured tumor cells with different CD44v6-expression (Fig. 2A). Results further confirmed the specific binding of AbD19384 to CD44v6. Binding and retention on CD44v6positive cells was in agreement with SPR measurements, with a slower off-rate for AbD19384 (Fig. 2A) than previously observed for the monovalent AbD15179 conjugate on cultured tumor cells $(7,14,18)$. The impact of labeling method (using chloramine-T or Iodogen) and radiohalogen $\left({ }^{125} \mathrm{I}\right.$ or $\left.{ }^{124} \mathrm{I}\right)$ was also assessed in vitro (Fig. 2B). No significant differences between the binding interactions of the different conjugates could be seen, demonstrating that the in vitro binding of the conjugates was not significantly influenced by labeling method or radionuclide in the present study.

In vivo specificity of ${ }^{125} \mathrm{I}-\mathrm{AbD} 19384$ was assessed by comparing uptake of ${ }^{125} \mathrm{I}-\mathrm{AbD} 19384$ in mice bearing both
MDA-MB-231 tumors (CD44v6 negative) and A431 tumors (high CD44v6 expression). Uptake in A431 tumors was shown to be significantly higher (four times higher) than the negative control tumors, confirming the specific binding of AbD19384 to CD44v6 also in vivo. A full biodistribution study of ${ }^{125} \mathrm{I}$-AbD19384 was performed in mice bearing H314 tumors (medium CD44v6-expression) and high CD44v6-expressing A431 tumors. Biodistribution data were favorable, with a pronounced clearance and decrease in activity over time for most organs, and tumor to blood ratios around five from $48 \mathrm{~h}$ p.i. (Fig. 3). The high uptake in thyroid, and initially high uptake in salivary glands and stomach, where $\mathrm{Na} / \mathrm{I}$ symporters are abundantly expressed, can probably be attributed to radiocatabolites of ${ }^{125} \mathrm{I}$ (19).

Biodistribution of ${ }^{125} \mathrm{I}-\mathrm{AbD} 19384$ was similar to the previously observed biodistribution of the monovalent ${ }^{125} \mathrm{I}$-AbD15179 fragment (18), with a maximum uptake in organs at the first time-point and fast clearance of the conjugate. As seen in Table I, kidney uptake was reduced with $\sim 50 \%$ for the bivalent fragment, whereas uptake in liver was only slightly increased. The reduced kidney uptake was expected, since AbD19384 displays a size above the renal threshold, whereas AbD15179 does not (20). However, since the bivalent Fab-dHLX construct is in equilibrium with a smaller fraction of the monovalent form, some monovalent tracers are expected to contribute to the biodistribution. It has previously been hypothesized that this may be an advantage in a tumor targeting setting, where monomers may penetrate tumors better and create effective dimers once at the target cells, due to the increased local concentration of the dimerization domains at the target site (9). In the present study, uptake in A431 tumors of monovalent ${ }^{125} \mathrm{I}-\mathrm{AbD} 15179$ (18) was comparable to that of bivalent ${ }^{125} \mathrm{I}-\mathrm{AbD} 19384$ at all time-points with no statistically significant difference (Table I). The bivalent fragment could have been expected to exhibit higher tumor uptake due to slower off-rate (21), as seen in the in vitro measurements (Fig. 2), but this was not confirmed in the in vivo studies. Thus, we conclude that both radio-iodinated AbD15179 and AbD19384 are suitable for CD44v6-specific tumor targeting 
in vivo, where AbD19384 can provide a lower kidney uptake than AbD15179 at similar tumor uptake levels. It is possible that tumor uptake for AbD19384 could be further increased by using a residualizing radiolabel, since a bivalent fragment may trigger an increased level of internalization, which could counteract a lower off-rate in the case of non-residualizing radioiodine label (22).

Due to the favorable biodistribution properties seen in the ${ }^{125} \mathrm{I}-\mathrm{AbD} 19384$ study, ${ }^{124} \mathrm{I}$ was selected for an imaging study and AbD19384 was subsequently labeled with ${ }^{124}$ I and assessed in PET/CT examinations, complemented with ex vivo biodistribution measurements (Figs. 4 and 5). Biodistribution measurements demonstrated more than two times higher uptake (calculated as \%ID/g) in high CD44v6-expressing A431 tumors compared to the low CD44v6-expressing UM-SCC74B tumors at all time-points. This was also shown by PET imaging, yielding higher contrast and higher uptake in A431 tumors at all three time-points. Uptake and elimination of ${ }^{124} \mathrm{I}-\mathrm{AbD} 19384$ was similar to ${ }^{125} \mathrm{I}-\mathrm{AbD} 19384$, with a maximum uptake in all organs at the first time-point and fast clearance of the conjugate. There was no significant difference in A431 tumor uptake between ${ }^{124}$ I-AbD19384 and ${ }^{125} \mathrm{I}$-AbD19384 conjugates. However, activity in blood was approximately two times higher for ${ }^{124} \mathrm{I}-\mathrm{AbD} 19384$ than for ${ }^{125} \mathrm{I}-\mathrm{AbD} 19384$ at $24 \mathrm{~h}$ p.i., resulting in a corresponding increase in the blood rich organs such as liver, spleen and kidneys for ${ }^{124} \mathrm{I}-\mathrm{AbD} 19384$. Uptake of ${ }^{124} \mathrm{I}$ in thyroid was successfully blocked by adding potassium iodide in the drinking water one day before measurements. Furthermore, radioactivity uptake in salivary glands and stomach was reduced with $\sim 2 / 3$ at $24 \mathrm{~h}$ p.i., most probably also a result of blocking. Even though the higher blood activity of ${ }^{124} \mathrm{I}-\mathrm{AbD} 19384$ resulted in lower tumor-to-blood ratios than obtained with ${ }^{125} \mathrm{I}$-AbD19384, A431 tumors were clearly visualized at all time-points, with the best contrast achieved at $48 \mathrm{~h}$ p.i. PET/CT images were in line with the biodistribution measurements, and tumor-to-blood ratios calculated from PET images were in very close agreement with the ex vivo data (Table II), further confirming the reliability of the non-invasive in vivo imaging technique.

In order to compare ${ }^{124} \mathrm{I}-\mathrm{AbD} 19384$ with standard imaging methods, ${ }^{18} \mathrm{~F}$-FDG was also employed as an imaging agent in PET/CT scans, and data were confirmed by ex vivo biodistribution measurements. There was no clear visualization of the tumors using ${ }^{18} \mathrm{~F}$-FDG. Furthermore, there was no clear discrimination between high CD44v6 expressing A431 tumors and low CD44v6 expressing UM-SCC74B tumors, since ${ }^{18} \mathrm{~F}-\mathrm{FDG}$ uptake is generally proportional to the metabolic activity in tissues and is not specific to tumor cells. High uptake of ${ }^{18} \mathrm{~F}$-FDG was observed in brown adipose tissue in the examinations (Fig. 5). This highlights one of the potential imaging pitfalls sometimes found also in clinical ${ }^{18} \mathrm{~F}$-FDG-PET during tumor staging (23) and emphasizes the need to complement this method with a more tumor-specific imaging technique.

In conclusion, our results describe for the first time a recombinant human Fab-dHLX fusion protein targeted to CD44v6, that possess high affinity, target specificity and potential for in vivo imaging of tumor biomarkers. This bivalent Fab antibody, engineered from monovalent Fab AbD15179 by subcloning of the Fab gene in fusion with a selfdimerizing helix-turn helix motif, was successfully produced and radioiodinated for molecular imaging of CD44v6 in vivo. Biodistribution and small-animal PET studies demonstrated that ${ }^{124} \mathrm{I}-\mathrm{AbD} 19384$ is a promising PET probe for imaging CD44v6 antigen expression in vivo. Furthermore, this proof-of-concept research established the feasibility of using recombinant Fab-dHLX constructs for in vivo imaging of tumor biomarkers. Additionally, Fab-dHLX constructs could be useful in the theranostic nanoplatform setting as a targeting ligand, and with further development of the other components of a successful theranostic agent it could also prove to be a valuable addition to personalized cancer treatment.

\section{Acknowledgements}

The authors would like to thank Jonas Stenberg for help with LigandTracer analysis and Veronika Asplund for help with ${ }^{18}$ F-FDG injections. The authors would like to acknowledge the Swedish Research Council, the Swedish Cancer Society, the Swedish Association for Medical Research and the Sweden America Foundation for kind support.

\section{References}

1. Chames P, Van Regenmortel M, Weiss E and Baty D: Therapeutic antibodies: Successes, limitations and hopes for the future. Br J Pharmacol 157: 220-233, 2009.

2. Olafsen T and Wu AM: Antibody vectors for imaging. Semin Nucl Med 40: 167-181, 2010.

3. Hoeben BA, Kaanders JH, Franssen GM, Troost EG, Rijken PF, Oosterwijk E, van Dongen GA, Oyen WJ, Boerman OC and Bussink J: PET of hypoxia with $89 \mathrm{Zr}$-labeled cG250-F(ab') in head and neck tumors. J Nucl Med 51: 1076-1083, 2010.

4. Willkomm P, Bender H, Bangard M, Decker P, Grünwald F and Biersack HJ: FDG PET and immunoscintigraphy with ${ }^{99 \mathrm{~m}} \mathrm{Tc}-$ labeled antibody fragments for detection of the recurrence of colorectal carcinoma. J Nucl Med 41: 1657-1663, 2000.

5. Khawli LA, Alauddin MM, Hu P and Epstein AL: Tumor targeting properties of indium-111 labeled genetically engineered $\mathrm{Fab}^{\prime}$ and $\mathrm{F}\left(\mathrm{ab}^{\prime}\right)_{2}$ constructs of chimeric tumor necrosis treatment (chTNT)-3 antibody. Cancer Biother Radiopharm 18: 931-940, 2003.

6. Bradbury AR, Sidhu S, Dübel S and McCafferty J: Beyond natural antibodies: The power of in vitro display technologies. Nat Biotechnol 29: 245-254, 2011.

7. Nilvebrant J, Kuku G, Björkelund $\mathrm{H}$ and Nestor M: Selection and in vitro characterization of human CD44v6-binding antibody fragments. Biotechnol Appl Biochem 59: 367-380, 2012.

8. Reichert JM and Dhimolea E: The future of antibodies as cancer drugs. Drug Discov Today 17: 954-963, 2012.

9. Pack P and Plückthun A: Miniantibodies: Use of amphipathic helices to produce functional, flexibly linked dimeric FV fragments with high avidity in Escherichia coli. Biochemistry 31: 1579-1584, 1992.

10. Eisenberg D, Wilcox W, Eshita SM, Pryciak PM, Ho SP and DeGrado WF: The design, synthesis, and crystallization of an alpha-helical peptide. Proteins 1: 16-22, 1986.

11. Orian-Rousseau V: CD44, a therapeutic target for metastasising tumours. Eur J Cancer 46: 1271-1277, 2010.

12. Pluckthun $A$ and Pack P: New protein engineering approaches to multivalent and bispecific antibody fragments. Immunotechnology 3: 83-105, 1997.

13. Nestor M, Sundström M, Anniko M and Tolmachev V: Effect of cetuximab in combination with alpha-radioimmunotherapy in cultured squamous cell carcinomas. Nucl Med Biol 38: 103-112, 2011.

14. Stenberg J, Spiegelberg D, Karlsson H and Nestor M: Choice of labeling and cell line influences interactions between the Fab fragment AbD15179 and its target antigen CD44v6. Nucl Med Biol 41: 140-147, 2014 
15. Hunter WM and Greenwood FC: Preparation of iodine-131 labelled human growth hormone of high specific activity. Nature 194: 495-496, 1962.

16. Larkin EA, Stiles BG and Ulrich RG: Inhibition of toxic shock by human monoclonal antibodies against staphylococcal enterotoxin B. PLoS One 5: e13253, 2010.

17. Kalinke U, Krebber A, Krebber C, Bucher E, Plückthun A, Zinkernagel RM and Hengartner H: Monovalent single-chain Fv fragments and bivalent miniantibodies bound to vesicular stomatitis virus protect against lethal infection. Eur J Immunol 26: 2801-2806, 1996.

18. Haylock AK, Spiegelberg D, Nilvebrant J, Sandström K and Nestor M: In vivo characterization of the novel CD44v6-targeting Fab fragment AbD15179 for molecular imaging of squamous cell carcinoma: A dual-isotope study. EJNMMI Res 4: 11, 2014.

19. Portulano C, Paroder-Belenitsky $\mathrm{M}$ and Carrasco N: The $\mathrm{Na}^{+} / \mathrm{I}$ symporter (NIS): Mechanism and medical impact. Endocr Rev 35: 106-149, 2014.
20. Holechek MJ: Glomerular filtration: an overview. Nephrol Nurs J 30: 285-290; quiz 291-282, 2003.

21. Rudnick SI and Adams GP: Affinity and avidity in antibodybased tumor targeting. Cancer Biother Radiopharm 24: 155-161, 2009.

22. Schreiber AB, Libermann TA, Lax I, Yarden Y and Schlessinger J: Biological role of epidermal growth factor-receptor clustering. Investigation with monoclonal anti-receptor antibodies. J Biol Chem 258: 846-853, 1983.

23. Truong MT, Erasmus JJ, Munden RF, Marom EM, Sabloff BS, Gladish GW, Podoloff DA and Macapinlac HA: Focal FDG uptake in mediastinal brown fat mimicking malignancy: A potential pitfall resolved on PET/CT. AJR Am J Roentgenol 183: 1127-1132, 2004. 